Int. Journal of Fracture manuscript No.

(will be inserted by the editor)

\title{
Fracture size effects from disordered lattice
}

\section{models}

\author{
Mikko J. Alava ${ }^{1}$, Phani K.V.V. Nukala ${ }^{2}$, Stefano Zapperi ${ }^{34}$ \\ ${ }^{1}$ Department of Engineering Physicsry of Physics, Helsinki University of Tech- \\ nology, FIN-02015 HUT, Finland \\ ${ }^{2}$ Computer Science and Mathematics Division, Oak Ridge National Laboratory, \\ Oak Ridge, TN 37831-6164, USA \\ ${ }^{3}$ CNR-INFM, S3, Dipartimento di Fisica, Università di Modena e Reggio Emilia, \\ Via G. Campi 213A, Modena, Italy \\ ${ }^{4}$ ISI Foundation, Viale S. Severo 65, 10133 Torino, Italy
}

Received: date / Revised version: date

\begin{abstract}
We study size effects in the fracture strength of notched disordered samples using numerical simulations of lattice models for fracture. In particular, we consider the random fuse model, the random spring model and the random beam model, which all give similar results. These allow us to establish and understand the crossover between a regime controlled by disorder-induced statistical effects and a stress-concentration controlled regime ruled by fracture mechanics. The crossover is described by a scaling law that accounts for the presence of fracture process zone which we quan-
\end{abstract}


tify by averaging over several disordered configurations of the model. The models allow to study the development of the fracture process zone as the load is increased and to express this in terms of crack resistance (R-curve).

\section{Introduction}

Understanding how materials break is a fundamental open problem of science and engineering. The difficulties stem from the non-trivial dependence of the fracture strength on the characteristic lengthscales of the samples, as already noted by Leonardo da Vinci, who measured the carrying-capacity of metal wires of varying length (1). He observed that the longer the wire, the less weight it could sustain. The reason for this behavior is rooted in the structural disorder present in the material: the strength is dominated by the weakest part (subvolume) of the sample and its distribution can in principle be computed using extreme value statistics (2). Longer wires have more weak parts and are thus bound to fail at smaller loads on average. The quantitative understanding of this statistical size effect is difficult, since the important low-strength tails of the strength probability distribution are not easy to sample and since the material properties are often history-dependent. For instance, in quasi-brittle materials such as concrete and many other composites, where sample failure is preceded by significant damage accumulation (3).

An important engineering scenario and a typical experimental setting is to study the size effect in the presence of a pre-existing flaw, a notch. 
Failure in this case is determined by the competition between deterministic effects, due to the stress enhancement created by notch, and the response of the disordered material around the defect to the stress concentration $(4 ;$; $)$. This includes the stochastic damage accumulation. The effect of disorder can be treated in an effective medium sense by defining a Fracture Process Zone (FPZ) around the crack tip. For quasi-brittle materials, the size of this FPZ may not be negligible compared to the system size. Conversely, for small notches failure is more influenced by the FPZ than the notch itself, and depends on statistical disorder effects. Experimentally, it has been demonstrated that the critical crack may nucleate and propagate far from the pre-existing notch (마) in that case.

The existing theories on the size effect start from linear elastic fracture mechanics (LEFM). Several formulations have been proposed in the literature and partly compared with experiments $(5 ; 7 ; 8 ; 99 ; 10 ; 11 ; 12)$. The problem is how to extend LEFM in the presence of disorder and concomitant damage.

In LEFM the Griffith's stability criterion or equation for the maximum stress the specimen can bear reads $\sigma_{c} \sim K_{c} / \sqrt{a_{0}}$. Here $a_{0}$ is the linear size of the crack and the critical stress intensity factor $K_{c} \sim \sqrt{E G_{c}}$ is a function of the fracture toughness $G_{c}$ and the elastic modulus $E$ (13). In the size-effect law proposed by Bazant for quasi-brittle materials $(5 ; 9 ; 10)$, the Griffith expression is generalized considering an additional lengthscale 
$\xi$ due to the presence of a FPZ

$$
\sigma_{c}=K_{c} / \sqrt{\xi+a_{0}}
$$

Equation (11) takes into account the both limits of a very large notch compared to the FPZ size and that of a very small notch. In the limit $\xi / a_{0} \ll 0$ one has an expression that follows the LEFM scaling, $\sigma_{c} \sim 1 / \sqrt{a_{0}}$. In the opposite limit of $a_{0} \rightarrow 0$, the average strength is taken to be constant. Eq. (11) has been shown to be in agreement with several experimental data sets (10). However, three fundamental questions can be asked: first, does Eq. (11) incorporate all the important effects? Second, what is the fracture toughness $G_{c}$ in the presence of disorder? Third, where does the FPZ scale $\xi$ originate and how does it depend again on the disorder?

In this work we clarify the role of the disorder in the failure of notched quasi-brittle specimens using extensive simulations of disordered lattice models for fracture (14). A brief account of the results for the RFM has been published in Ref. (15). In more detail, we consider the random fuse model (RFM), the random spring model (RSM) and the random beam model (RBM). We consider the failure of notched disordered samples and provide a microscopic justification of Eq. (11). Studying the size scaling of strength by extensive numerical simulations is a difficult task due to the different length scales involved and the need of significant statistical averaging. We vary the disorder, which we model as a locally varying random failure threshold, and show that it plays a crucial role in determining the size effect. In particular, a lengthscale $\xi$ emerges from the simulations and 
can be shown to be directly related to the FPZ size. Finally, for notch sizes smaller than a critical length $a_{c}$, we observe a cross-over to the inherent, sample-size dependent strength of the unnotched sample. We outline the scaling theory to account for these effects. We show that the RFM results are confirmed in the RSM and the RBM. We also study the growth of the FPZ, showing that it is independent of the notch size $a_{0}$ and the system size $L$.

\section{Models}

\subsection{Random fuse model}

In the RFM (16), we consider a triangular lattice of linear size $L$ with a central notch of length $a_{0}$. Each fuse has the same conductance and a random breaking threshold $t$. This represents a locally varying fracture toughness/strength. The $t$ lie between 0 and 1 , with a cumulative distribution $P(t)=t^{1 / D}$, where $D$ represents a quantitative measure of disorder. The larger $D$ is, the stronger the disorder. The burning of a fuse occurs irreversibly, whenever the electrical current in the fuse exceeds breaking threshold $t$ of the fuse. Periodic boundary conditions are imposed in the horizontal direction to simulate an infinite system and a constant voltage difference, $V$, is applied between the top and the bottom of lattice system bus bars. Numerically, a unit voltage difference, $V=1$, is set between the bus bars and the Kirchhoff equations are solved to determine the current flowing in each of the fuses. Subsequently, for each fuse $j$, the ratio between 
the current $i_{j}$ and the breaking threshold $t_{j}$ is evaluated, and the bond $j_{c}$ having the largest value, $\max _{j} \frac{i_{j}}{t_{j}}$, is irreversibly removed (burnt). The current is redistributed instantaneously after a fuse is burnt implying that the current relaxation in the lattice system is much faster than the breaking of a fuse. Each time a fuse is burnt, it is necessary to re-calculate the current redistribution in the lattice to determine the subsequent breaking of a bond. The process of breaking of a bond, one at a time, is repeated until the lattice system fails completely. In the present simulations, we have considered various notch sizes for $L=64,128,192,256,320$ and $D=0.1,0.3,0.5,0.6,0.75$.

\subsection{Random spring model}

In the RSM, we consider a triangular lattice with nodes connected by linear springs with unit stiffness $(17 ; 18 ; 19 ; 20 ; 21)$. As for the RFM, the bond breaking thresholds, $t$, are randomly distributed based on a thresholds cumulative probability distribution, $P(t)=t^{1 / D}$ for $t \in[0,1]$. The bond breaks irreversibly, whenever the force in the spring exceeds the breaking threshold force value, $t$, of the spring. Periodic boundary conditions are imposed in the horizontal direction and a displacement difference is applied between the top and the bottom of the lattice system. Numerically, a unit displacement, $\Delta=1$, is applied at the top of the lattice system and the equilibrium equations are solved to determine the force in each of the springs. Subsequently, for each bond $j$, the ratio between the force $f_{j}$ and the breaking threshold $t_{j}$ is evaluated, and the bond $j_{c}$ having the largest value, $\max _{j} \frac{f_{j}}{t_{j}}$, 
is irreversibly removed. The forces are redistributed instantaneously after a bond is broken implying that the stress relaxation in the lattice system is much faster than the breaking of a bond. Each time a bond is broken, it is necessary to re-equilibrate the lattice system in order to determine the subsequent breaking of a bond. The process of breaking of a bond, one at a time, is repeated until the lattice system falls apart. For the RSM, we consider a triangular lattice network of size $L$ with a notch of size $a_{0}$. In the present simulations, we have considered various notch sizes for $L=128,256$ and $D=0.5,0.6$.

\subsection{Random beam model}

In the random thresholds beam model (RBM) $(22 ; 23)$, we consider a twodimensional triangle lattice system of linear size $L$. The vectorial RBM has three degrees of freedom ( $\mathrm{x}$-translation $u, \mathrm{y}$-translation $v$, and a rotation $\theta$ about $\mathrm{z}$ axis) at each of the lattice nodes (sites), and each of the bonds (beams) in the lattice connects two nearest neighbor nodes. We assume that the beams are connected rigidly at each of the nodes such that the angle between any two beams connected at a node remains unaltered during the deformation process. These nodal displacements and rotations introduce conjugate forces and bending moments in the beam members. Using Tim-

oshenko beam theory (24), which includes shear deformations of the beam cross-section in addition to the usual axial deformation of cross-sections, the local stiffness matrix for a beam element that relates the local nodal 
displacements and rotations to local nodal forces and bending moments in the beam's local coordinate system is given by

$$
\mathbf{K}_{\text {local }}=\left[\begin{array}{cccccc}
\frac{E A}{\ell_{b}} & 0 & 0 & -\frac{E A}{\ell_{b}} & 0 & 0 \\
\frac{12 E I}{(1+\alpha) \ell_{b}^{3}} & \frac{6 E I}{(1+\alpha) \ell_{b}^{2}} & 0 & -\frac{12 E I}{(1+\alpha) \ell_{b}^{3}} & \frac{6 E I}{(1+\alpha) \ell_{b}^{2}} \\
& \frac{(4+\alpha) E I}{(1+\alpha) \ell_{b}} & 0 & -\frac{6 E I}{(1+\alpha) \ell_{b}^{2}} & \frac{(2-\alpha) E I}{(1+\alpha) \ell_{b}} \\
& & \frac{E A}{\ell_{b}} & 0 & 0 \\
S Y M & & & \frac{12 E I}{(1+\alpha) \ell_{b}^{3}} & -\frac{6 E I}{(1+\alpha) \ell_{b}^{2}} \\
& & & & & \frac{(4+\alpha) E I}{(1+\alpha) \ell_{b}}
\end{array}\right]
$$

where $E$ is the Young's modulus, $G$ is the shear modulus, $A$ is the beam cross-sectional area, $I$ is the moment of inertia of beam cross-section, $\ell_{b}$ is the length of the beam, and $\alpha=\frac{12 E I}{G A \ell_{b}^{2}}$ is the shear correction factor, which denotes the ratio of bending stiffness to the shear stiffness. If shear deformation of beam cross-section is negligible, then $\alpha=0$ and the Timoshenko beam theory reduces to Euler-Bernoulli beam theory. Equation 2 presents a relation between local nodal displacements and rotations $\mathbf{d}_{\ell}=\left(u_{l i}, v_{l i}, \theta_{l i}, u_{l j}, v_{l j}, \theta_{l j}\right)^{T}$ and local forces and moments $\mathbf{F}_{\ell}=$ $\left(F_{l i}, V_{l i}, M_{l i}, F_{l j}, V_{l j}, M_{l j}\right)^{T}$. In this setting, the subscript $l$ refers to local quantities, the superscript $T$ represents transpose of a vector or a matrix, $i$ and $j$ refer to $i$-th and $j$-th nodes of the beam, and $F, V$, and $M$ refer to axial force, shear force, and bending moments respectively.

Equilibration of the lattice system is achieved by first transforming these local quantities $\left(\mathbf{d}_{\ell}\right.$ and $\left.\mathbf{F}_{\ell}\right)$ into global quantities $\mathbf{d}=\left(u_{i}, v_{i}, \theta_{i}, u_{j}, v_{j}, \theta_{j}\right)^{T}$ and $\mathbf{F}=\left(F_{i}, V_{i}, M_{i}, F_{j}, V_{j}, M_{j}\right)^{T}$ through a coordinate transformation $\mathbf{T}$ 
such that $\mathbf{d}_{\ell}=\mathbf{T d}, \mathbf{F}_{\ell}=\mathbf{T F}$, and $\mathbf{K}=\mathbf{T}^{T} \mathbf{K}_{\text {local }} \mathbf{T}$, and then satisfying equilibrium equations at each node such that

$$
\Sigma_{<i j>} F_{x}=0 ; \quad \Sigma_{<i j>} F_{y}=0 ; \quad \Sigma_{<i j>} M=0
$$

where $\Sigma_{<i j\rangle}$ implies that the summation is carried over all the intact bonds $<i j>$ joining at node $i$. In the above discussion, the transformation matrix $\mathbf{T}$ is given by

$$
\mathbf{T}=\left[\begin{array}{ll}
\mathrm{Q} & 0 \\
0 & \mathrm{Q}
\end{array}\right]
$$

where

$$
\mathbf{Q}=\left[\begin{array}{ccc}
c & s & 0 \\
-s & c & 0 \\
0 & 0 & 1
\end{array}\right]
$$

and $c=\cos (\beta), s=\sin (\beta)$ refer to the direction cosines of the beam with $\beta$ representing the angle between the beam axis and the $x$-direction.

In the present simulation, we start with a notched lattice system with beams having unit length, unit square cross-section and Young's modulus $E=1$. This results in a unit axial stiffness $\left(E A / \ell_{b}=1\right)$ and bending stiffness $\left(12 E I / \ell_{b}^{3}=1\right)$ for each of the beams in the lattice system. Since the beam can deform in two independent deformation modes (axial and bending), we assume randomly distributed bond breaking axial and bending thresholds, $t_{a}$ and $t_{b}$, based on thresholds cumulative probability distributions, $P_{a}\left(t_{a}\right)$ and $P_{b}\left(t_{b}\right)$ respectively. As in the other models, the cumulative distributions are defined as $P(t)=t^{1 / D}$ in $[0,1]$. 
The failure criterion for a beam is defined through an axial force and bending moment interaction equation (similar to von-Mises criterion in metal plasticity) given by

$$
r \equiv\left(\frac{F}{t_{a}}\right)^{2}+\frac{\max \left(\left|M_{i}\right|,\left|M_{j}\right|\right)}{t_{b}}=1
$$

The beam breaks irreversibly, whenever the failure criterion $r \geq 1$. Periodic boundary conditions are imposed in the horizontal direction and a constant unit displacement difference is applied between the top and the bottom of lattice system.

Numerically, a unit displacement, $\Delta=1$, is applied at the top of the lattice system and the equilibrium equations (Eq. 3) are solved to determine the force in each of the springs. Solution of Eq. 3 results in global displacements and rotations $\mathbf{d}$, using which the local displacements $\mathbf{d}_{\ell}=\mathbf{T} \mathbf{d}$ and the local forces $\mathbf{F}_{\ell}=\mathbf{K}_{\text {local }} \mathbf{d}_{\ell}$ are computed for each of the intact beams. Subsequently, for each bond $k$ with nodes $i$ and $j$, the quantities $a_{k}=\left(\frac{F}{t_{a}}\right)^{2}$ and $b_{k}=\frac{\max \left(\left|M_{i}\right|,\left|M_{j}\right|\right)}{t_{b}}$ are evaluated, and the bond $k_{c}$ having the smallest value,

$$
r_{k}=\frac{-b_{k}+\sqrt{b_{k}^{2}+4 a_{k}}}{2 a_{k}}
$$

is irreversibly removed (When $a_{k}=0$, then $r_{k}=\frac{1}{b_{k}}$ ). The forces are redistributed instantaneously after a bond is broken implying as in the other models that the stress relaxation in the lattice system is much faster than the breaking of a bond. Each time a bond is broken, it is necessary to reequilibrate the lattice system in order to determine the subsequent breaking 

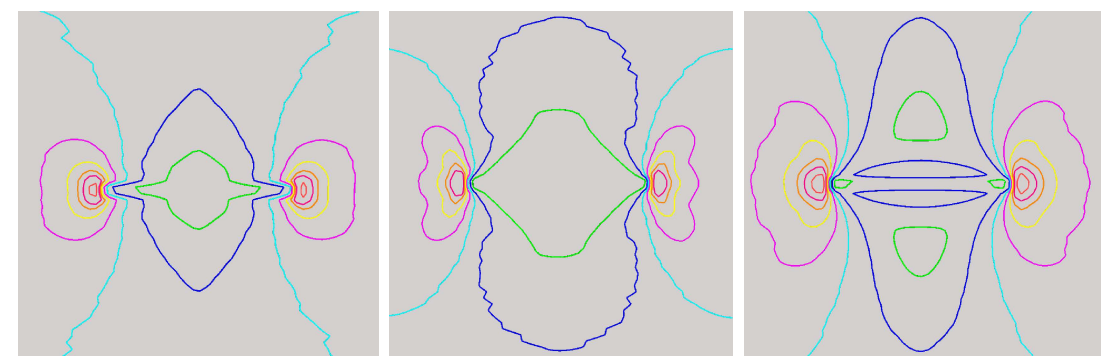

Fig. 1 The stress concentration profiles for the RFM (left), the RSM (center) and the RBM (right).

of a bond. The process of breaking of a bond, one at a time, is repeated until the lattice system falls apart.

\section{Strength and size effects}

We perform numerical simulations of the models discussed above, concentrating on the failure strength. Notice that the three model differ mainly in the way stress is redistributed. To illustrate this point, we report in Fig. 1 the stress concentration profiles in a triangular lattice $(L=512)$ with a notch of size $a_{0}=16$. Although the angular distribution of the stress profiles differs, the way stress decay from the crack tip is very similar, approaching for large $L$ the $1 / \sqrt{r}$ decay expected from the theory of elasticity.

In order to obtain reliable results, the strength should be averaged over several realizations of the disorder. In the present simulations, we have used a minimum of $N_{r}=200$ realizations and in some cases up to $N_{r}=8000$ realizations. Fig. 2 a reports the strength $\sigma_{c}$, averaged over different configurations, with varying $a_{0}, D$, and $L$ for the RFM. The most instructive 
way of plotting is to consider the inverted square strength, $1 / \sigma_{c}^{2}$. Assuming Eq. (1), $1 / \sigma_{c}^{2}$ should become a linear function of $a_{0}$ for large enough notches. Plotting the data in this way in Fig. 20 shows that for $a_{0} \gg 1$, the scaling of Eq. (11) is recovered asymptotically. Extrapolating the linear part towards $a_{0}=0$, we can define a disorder-dependent intercept $\xi(D)$, that should be related to the FPZ size. Furthermore, the slope of the linear part of the data $\left(1 / K_{c}^{2}(D)\right)$ is also disorder-dependent, which implies a disorder-dependent fracture toughness $G_{c}(D)$. Finally, a careful observation reveals that for small $a_{0}$ less than a critical crack size $a_{c}$, the strength scaling crosses over from a stress concentration dominated LEFM scaling [Eq. (10] to a disorder dominated scaling (see Fig. 3). That is, for $a_{0} \ll a_{c}$, the strength scaling deviates significantly from Eq. (11) and saturates to a value that depends on disorder and the sample size, $\sigma_{c}(L, D)$. In particular, the strength of the unnotched system (for $a_{0}=0$ ) is finite and is smaller than the LEFM limit $K_{c} / \sqrt{\xi}$ given by Eq. (11). In Fig. 3, we present a comparison between RFM, RSM and the RBM. The general features of the strength are the same in the three models, indicating that only the $1 / \sqrt{r}$ decay of stress concentration is relevant for the size effect, while the precise angular dependence of stress concentration around a notch is not important.

In Ref. (15) we presented a scaling theory that extends the earlier scaling law given by Eq. (1) beyond its actual regime of validity. A correct scaling expression has to accommodate the three separate phenomena visibile in Fig. 2, for small notches, the dominance of statistical effects that 
dictate $\sigma_{c}(L, D)$, the cross-over to the LEFM-like regime, and then finally an Equation (1) like scaling at large $a_{0}$.

The cross-over takes place at a scale $a_{c}$ above which $\sigma_{c}$ follows Eq. (1). For small notches, $a_{0} \ll a_{c}$ the strength is determined by extremal statistics as is appropriate in the limit $a_{0} \rightarrow 0(14)$. Then one expects to see a weak size effect, typically logarithmic in $L$. In real materials, the scaling will depend on the damage accumulation and the defect populations that exist in the specimens. $\sigma_{c}(L, D)$ is not a constant however, as Eq. (1) would predict, and deviates significantly from the LEFM-based theory, which would in general predict that the samples are weaker than their actual strength $\sigma_{c}(L, D)$.

The location of the cross-over (notch size) $a_{c}$ follows by equating the strength prediction of Eq. (1) and the scaling of notchless specimes, $1 / \sigma(L, D)^{2} \simeq$ $\left(a_{c}+\xi\right) / K_{c}^{2}$. An appropriate scaling theory, valid for all $a_{0}$, is then

$$
\frac{K_{c}^{2}}{\sigma_{c}^{2}}=\xi+a_{0} f\left(a_{c} / a_{0}\right)
$$

where the statistical physics -like scaling function $f(y)$ has the limits

$$
f(y) \simeq \begin{cases}1 \text { if } & y \ll 1 \\ y \text { if } & y \gg 1\end{cases}
$$

Thus we have for the cross-over scale

$$
a_{c} \simeq\left(K_{c}(D) / \sigma_{c}(L, D)\right)^{2}-\xi(D) .
$$

For $a_{0}>a_{c}$, fracture is governed by LEFM and a scaling of the kind of Eq. (11) is recovered. The effect of disorder, according to the scaling theory, 
is incorporated in the three parameters $\left.\sigma_{c}(L, D)\right), K_{c}(D)$, and $\xi(D)$. In the following, we first discuss the first two parameters and return to $\xi$ below in much more detail. Qualitatively (since the behaviour of $\sigma_{c}(L, D)$ is an independent issue entirely), the effect of changing disorder strength for a fixed $L$ can be seen as follows. For stronger disorder, the cross-over will take place at larger $a_{c}$, since the stress concentration of the notch will be screened (as we demonstrate below). At fixed disorder, $a_{c}$ increases with $L$ since notchless specimens get weaker. The fracture toughness $G_{c}$ (since in the models $E=1$ ) seems in our simulations to be proportional to the average model element strength at weak disorder at least, and perhaps gets reduced with strong disorder (15). More numerical work in this direction might be interesting.

\section{The fracture process zone}

Our numerical simulations allow to monitor the damage evolution prior to failure and can thus be used to study the development of the FPZ. For a single realization of the disorder, we only see diffuse damage up to the peak load, and it is difficult to determine the size of the FPZ. On the other hand, the FPZ can be clearly measured after averaging the damage over several realizations of the disorder. Hence, the size of FPZ should thus be considered in statistical terms as the region around the crack tip where damage is most likely to occur. Considering for simplification a projection of the average damage along the notch main axis direction, we obtain a profile 

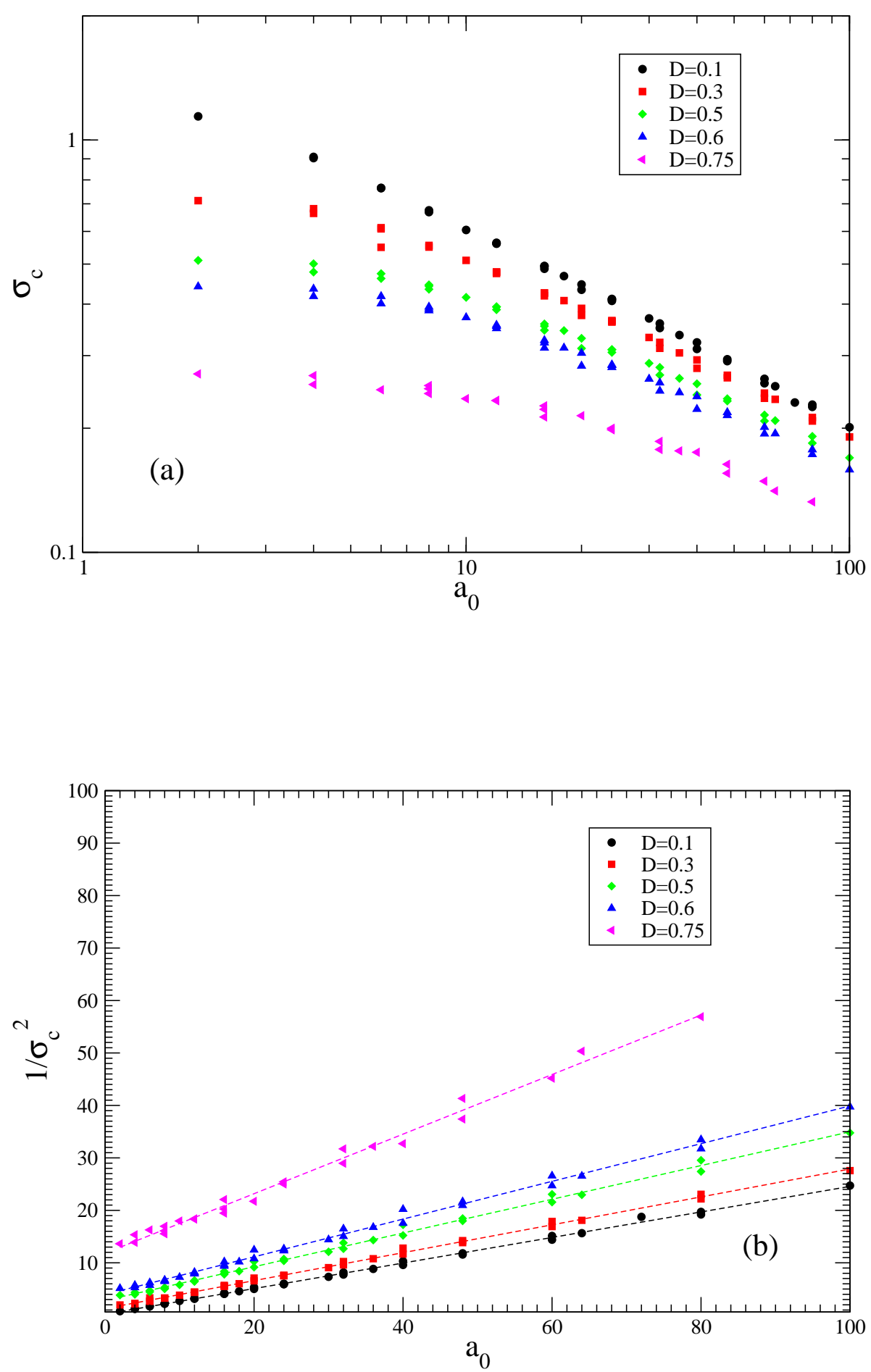

Fig. 2 a): The strength in the RFM for several disorders $D$, notch sizes $a_{0}$ and system sizes $L$. b): a scaling plot of the data according to Eq. (11). 


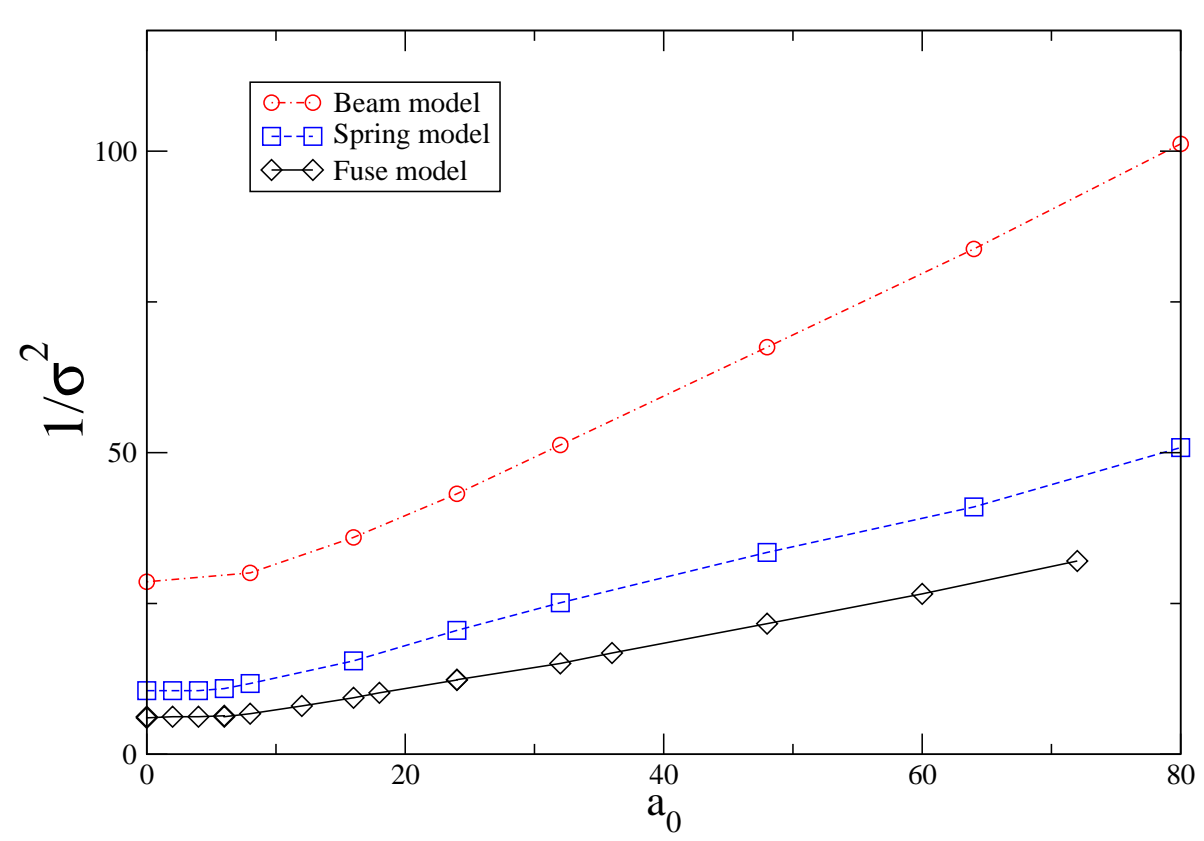

Fig. 3 A scaling plot of the strength according to Eq. (1) for the RFM (for $L=192, D=0.6)$, the $\operatorname{RSM}(L=256 D=0.5)$ and the $\operatorname{RBM}(L=256 D=1.0)$ models. The qualitative features of the data for different system sizes and disorders are the same in all the three models.

that is decaying exponentially towards a homogeneous background value: $d(x)=A+B \exp \left(-2 x / \xi_{F P Z}\right)$ (see Fig. 4). The factor 2 in the exponential comes from the fact that in our geometry the FPZ extends from the two edges of the notch. We have analyzed the data for different values of $a_{0}$ and $L$ in order to check that the profiles do not depend on $L$ and on $a_{0}$, as long as this is not too far from $a_{c}$. For $a_{0}<<a_{c}$ we naturally do not expect to see such a "damage cloud" around the original defect. However, it seems likely 
that one could measure $\xi$ around the most critical microcrack. Recall that in this regime one expects the strength to saturate at $\sigma_{c}=\sqrt{K_{c} /\left(a_{c}+\xi\right)}$.

Notice that the LEFM stress intensity factor would indicate a $1 / \sqrt{r}$ like divergence of the stress at the crack tip. It is evident that the observed exponential decay of damage profile $d$ is in contrast to a $1 / \sqrt{r}$-like decay and should be naturally interpreted as a screening of the crack tip caused by the disorder. In fact, the FPZ size $\xi_{F P Z}$ depends on the disorder strength $D$ as shown in Fig. 5 . The data can be roughly described by a power law relation $\xi_{F P Z} \sim D^{3 / 2}$. As discussed in Ref. (15), if we plot the fracture process size $\xi_{F P Z}(D)$ against the intrinsic scale $\xi$ resulting from the fits of the strength data to Eq. (11), we obtain a linear relation. Hence, we can conclude that $\xi$ is indeed a direct measure of the FPZ size. Fig. 6 reports a comparison of the damage profiles in RFM, RSM and RBM. It can be seen that the results are qualitatively similar for all the three models considered. The value of the FPZ size $\xi_{F P Z}$, however, differs slightly for the for the three models.

The FPZ progressively develops before the peak load by damage accumulation. To visualize this process, we have computed damage profiles at different values of the applied stress. One can then obtain the FPZ size $\xi_{F P Z}(D)$ as a function of the stress. As can be seen in Fig. 7 , there is a gradual increase of $\xi_{F P Z}(D)$ with stress. This growth relates to the R-curve of the material (11) which is usually defined in terms of the elastic energy released due to crack growth $G \equiv \partial U / \partial a(4)$. In the RFM model, we can 
derive $G$ from the lattice "elastic" energy $U=I^{2} /(2 \Sigma)$, where $\Sigma$ is the conductivity, as

$$
G \approx \frac{\Delta U}{\Delta a}=\frac{I^{2}}{2 \Sigma^{2}} \frac{\Delta \Sigma}{\Delta a}
$$

where $\Delta \Sigma=\left(\Sigma_{0}-\Sigma\right)$ is the conductivity change after the crack has extended by $\Delta a$ such that $a=a_{0}+\Delta a$, and $\Sigma_{0}$ is the initial conductivity. We report the R-curve for different values of disorder in Fig. 8. The data is shown for the current values $I \in\left[I_{c} / 2,\right]$ in which $\xi(I)$ can be extracted from the damage profiles with a reliable accuracy. In this regime the Rcurves show in general two behaviors: an initial rapid increase due to the accumulating damage that changes the average conductivity, followed by a saturation as the FPZ starts to increase even more rapidly. The conductivity change is a mean-field phenomenon that accounts for the total damage in the system, proportional to $D(I)$, and thus to the failure thresholds $P\left(i_{c}\right)$. The growth of $\xi(I)$ is not expected to be so simply related to $D$. It is interesting to note that as a result the R-curves for various disorders overlap in the manner depicted in Fig. 8 , The size-dependence of these R-curves would be expected to be negligible as long as the strength is governed by Eq. (1).

\section{Conclusion}

We have resorted to simulations of statistical fracture models to analyze the problem of the size-effect in the failure of quasi-brittle materials. For large

notches, the simulations recover the expression based on LEFM $(5 ; 9 ; 10)$ and allow to relate the effective FPZ size $\xi$ to the actual average damage 
Fr:
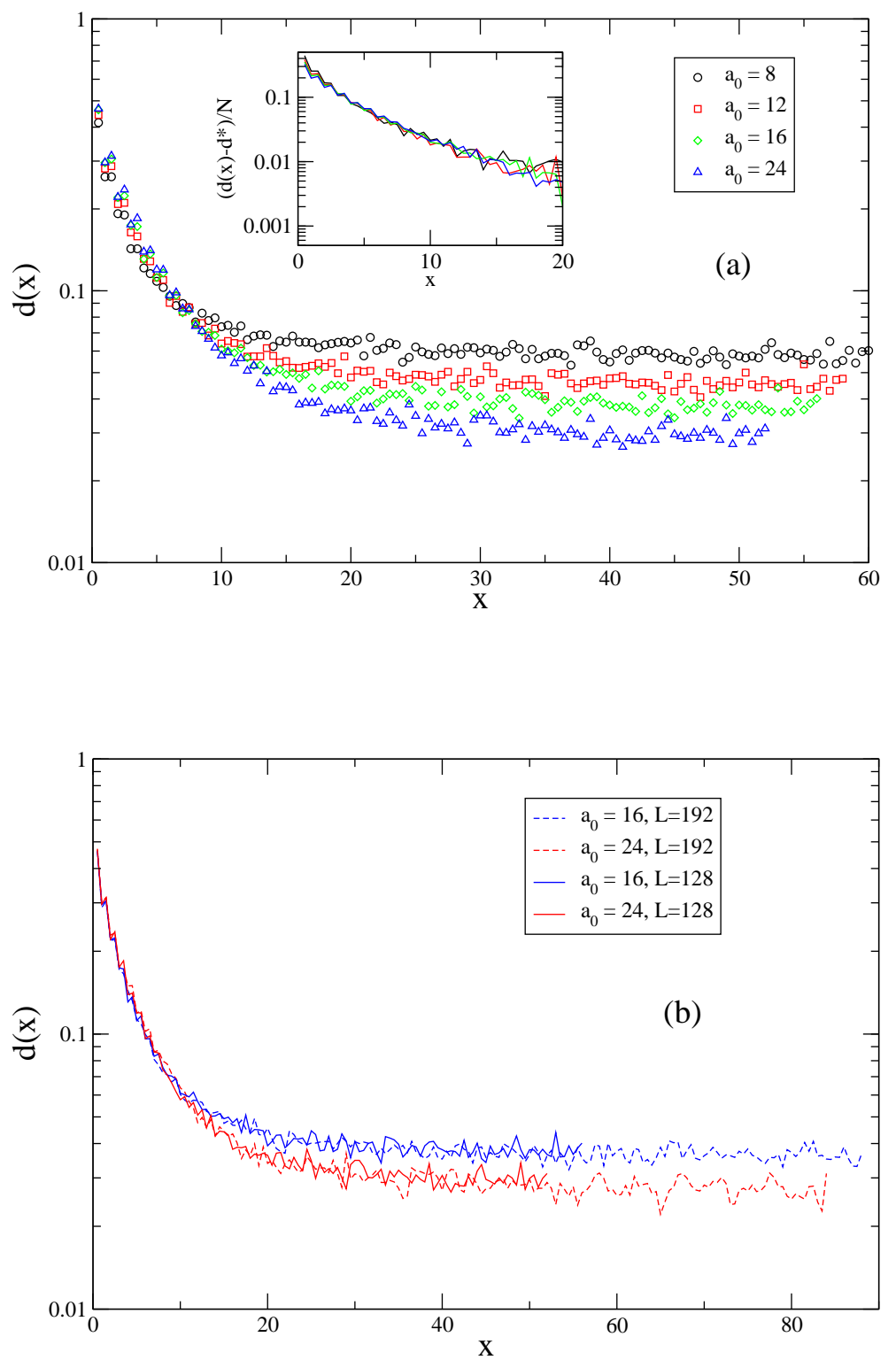

Fig. 4 a): Damage profiles along the crack axis for various notch sizes $a_{0}$. Damage profiles follow an exponential decay on a uniform damage background, i.e., $d(x)=$ $A+B \exp \left(-2 x / \xi_{F P Z}\right)$, where $A$ and $B$ are constants and $x$ is the distance from the crack tip along the crack axis. In order to show that $\xi_{F P Z}$ is independent on $a_{0}$, we report in the inset the profiles after subtracting the background and normalizing so that the curves superimpose. b) Damage profiles for two different lattice sizes $L$ and two different notch sizes $a_{0}$. The profiles do not depend on $L$. 


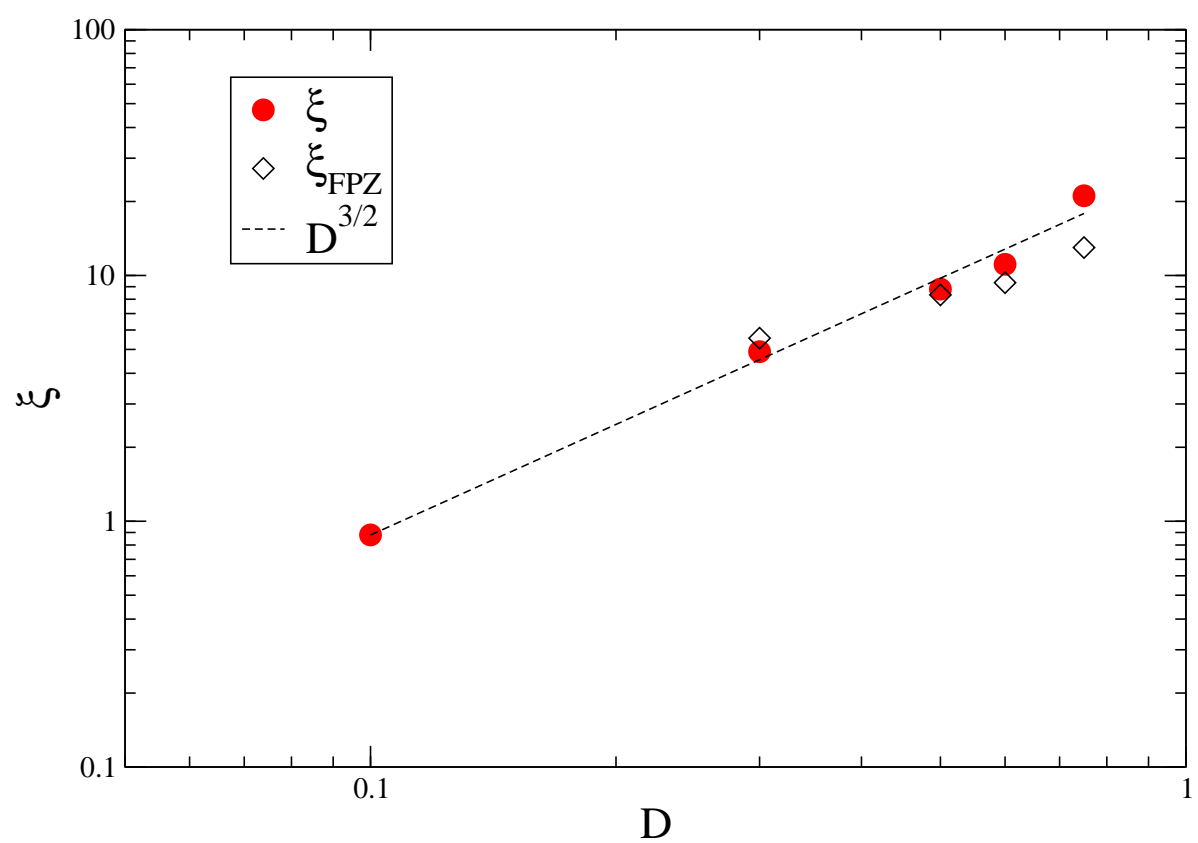

Fig. 5 The dependence of the FPZ size on disorder for the RFM ( $L=128$, $\left.a_{0}=16\right)$. Estimates from the strength $(\xi)$ and from damage profiles $\xi_{F P Z}$ are similar. We could not obtain reliable estimates from damage profiles for very weak disorder $(D=0.1)$.

profiles. As the notch size is decreased we observe a crossover at a novel scale $a_{c}$ to a disorder-dominated size-dependent regime that is not described by LEFM and is furthermore seen in experiments (15). All the regimes are summarized in a generalized scaling expression for the strength of disordered media.

Several interesting future questions remain, like theoretical computations of parameters such as $a_{c}, K_{c}$, and the detailed understanding of the origin and shape of the statistical FPZ. These would be in particular im- 


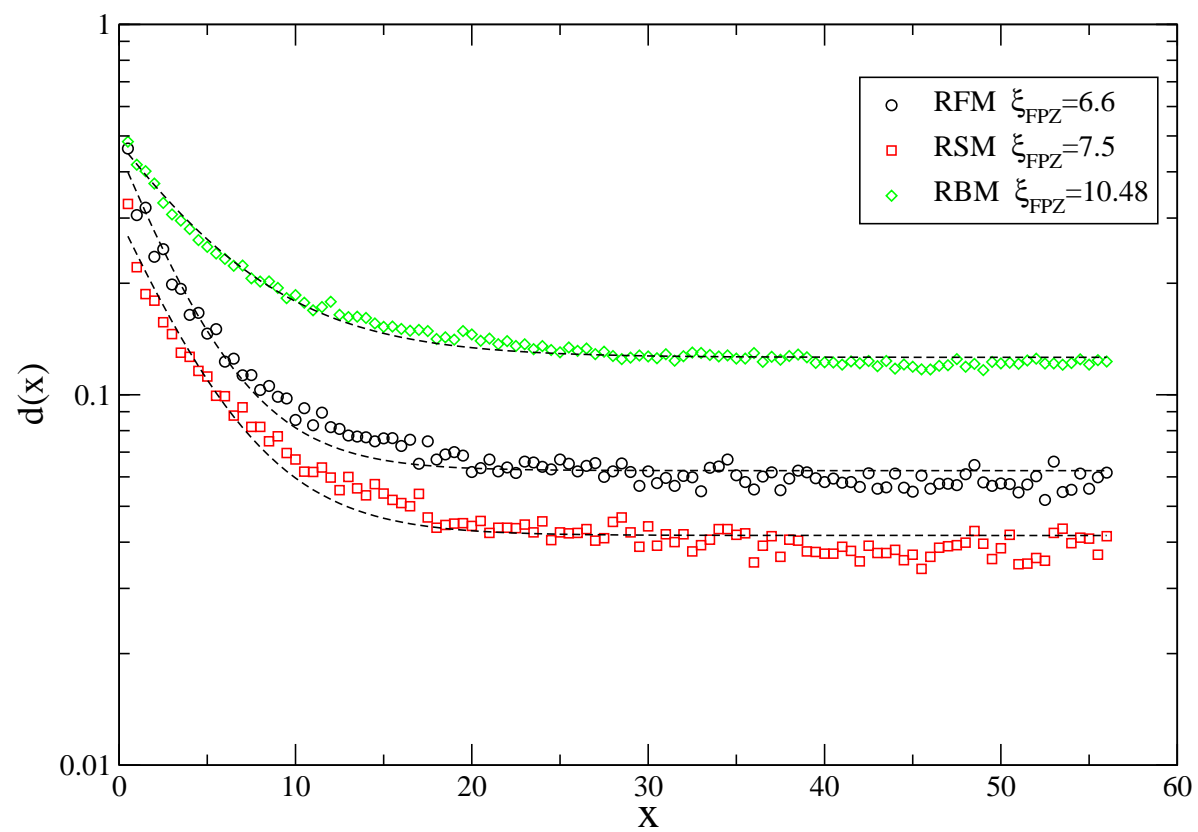

Fig. 6 A comparison of the damage profiles measured in RFM, RSM and RBM using system size $L=128$, disorder $D=0.6$, and an initial notch size $a_{0}=16$. Two thousand samples are used for averaging the damage profiles. The result show that while the profiles are qualitatively similar $\xi_{F P Z}$ and the damage backgound differ.

portant in order to help to achieve practical predictions. Recall our results have shown, that all such parameters are dependent on disorder, which in the models used translates into the damage accumulated at a given local stress. This quantity is evidently hard to access experimentally, but is unfortunately theoretically necessary. For such reasons, it would be relevant to investigate three dimensional systems and possibly look at other kinds of disorder (eg. locally varying elastic moduli). 


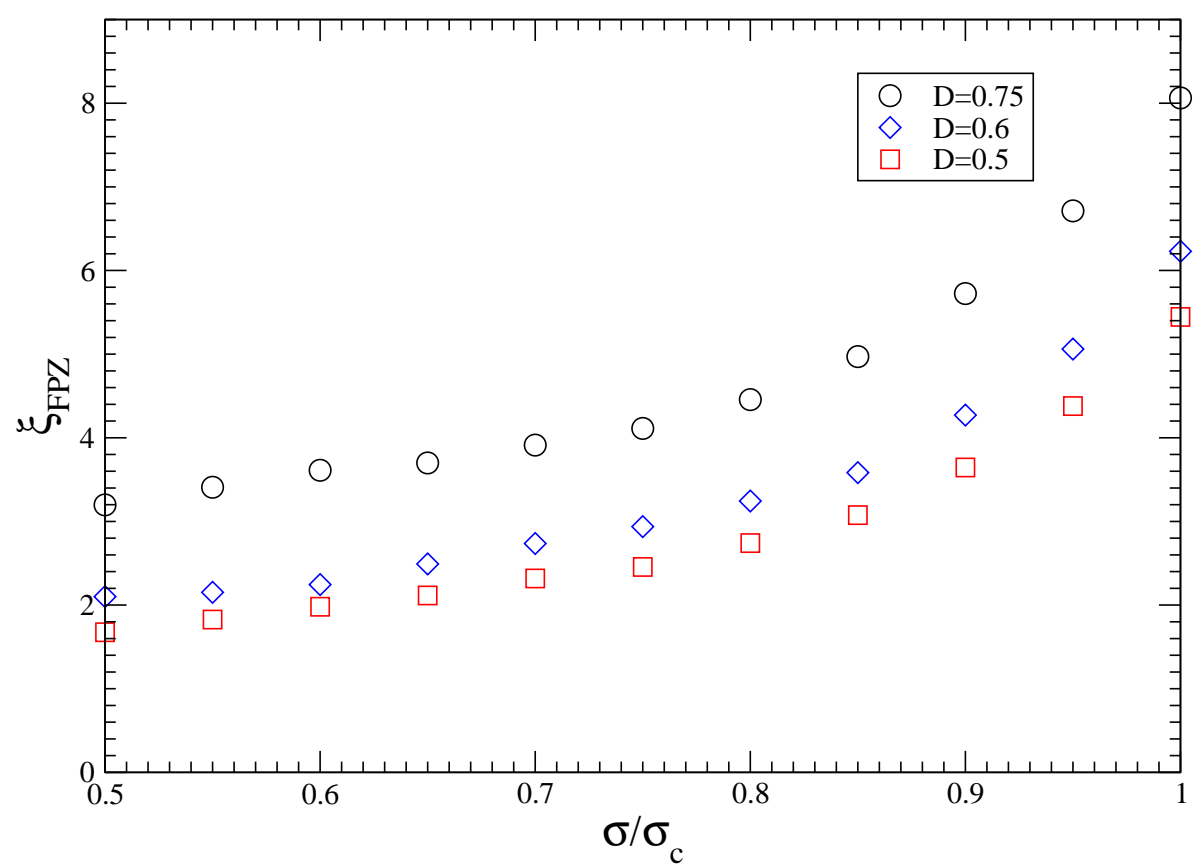

Fig. 7 The FPZ size as a function of the applied stress normalized by the peak stress as obtained from damage profiles. Data are for RFM for different values of disorder $\left(a_{0}=16, L=128\right)$.

Acknowledgments - MJA would like to acknowledge the support of the Center of Excellence -program of the Academy of Finland. MJA and SZ gratefully thank the financial support of the European Commissions NEST Pathfinder programme TRIGS under contract NEST-2005-PATHCOM-043386. PKKVN acknowledges support from Mathematical, Information and Computational Sciences Division, Office of Advanced Scientific Computing Research, U.S. Department of Energy under contract number DE-AC05-00OR22725 with UT-Battelle, LLC. PKKVN also acknowledges 


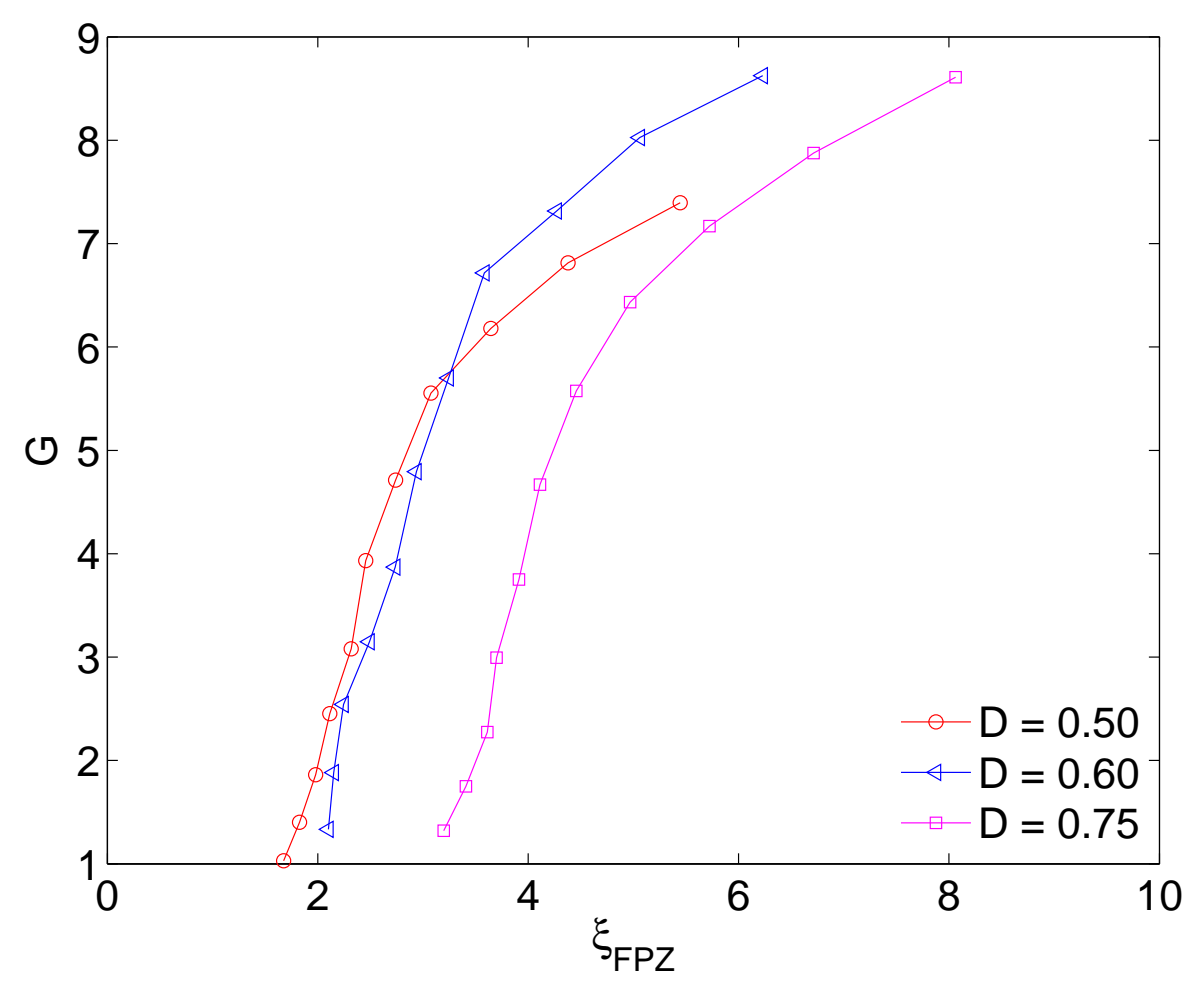

Fig. 8 The R curve of the RFM for different values of the disorder D.

the use of IBM BG/L resources made available to him at Argonne National Laboratory through INCITE.

\section{References}

1. L. da Vinci, I libri di Meccanica (Hoepli Milano 1940).

2. E. J. Gumbel, Statistics of Extremes (Columbia University Press, New York, 2004).

3. J. G. M. van Mier, Fracture Processes of Concrete (CRC Press, Boca Raton, USA, 1996). 
4. Z. P. Bazant and J. Planas, Fracture and Size Effect in Concrete and Other Quasibrittle Materials (CRC Press, Boca Raton, USA, 1997).

5. Z. P. Bazant, Arch. Appl. Mech. 69, 703 (1999).

6. J. Rosti, L. I. Salminen, E. T. Seppälä, M. J. Alava, and K. J. Niskanen, Eur. Phys. J. B 19, 259 (2001).

7. X. Z. Hu and F. Wittmann, Mat. Struct. 25, 319 (1992).

8. B. Karihaloo, Int. J. Fracture 95, 379 (1999).

9. Z. P. Bazant, International Journal of Solids and Structures 37, 307 (2000).

10. Z. P. Bazant, PNAS 101, 13400 (2004).

11. S. Morel, J. Schmittbuhl, E. Bouchaud, and G. Valentin, Physical Review Letters 85, 1678 (2000).

12. S. Morel, E. Bouchaud, and G. Valentin, Phys. Rev. B 65, 104101 (2002).

13. A. A. Griffith, Trans. Roy. Soc. (london) A 221, 163 (1920).

14. M. J. Alava, P. Nukala, and S. Zapperi, Adv. Phys. 55, 349 (2006).

15. M. J. Alava, P. Nukala, and S. Zapperi, Phys. Rev. Lett. 100, 055502 (2008).

16. L. de Arcangelis, S. Redner, and H. J. Herrmann, Journal of Physics (Paris) Letters 46(13), 585 (1985).

17. M. Sahimi and J. D. Goddard, Physical Review B 33, 7848 (1986).

18. A. Hansen, S. Roux, and H. J. Herrmann, J. Physique 50, 733 (1989).

19. S. Arbabi and M. Sahimi, Physical Review B 47(2), 695 (1993). 
20. M. Sahimi and S. Arbabi, Physical Review B 47(2), 703 (1993).

21. P. K. V. V. Nukala, S. Zapperi, and S. Simunovic, Phys. Rev. E 71, 066106 (2005).

22. S. Roux and E. Guyon, J. Physique Lett. 46, L999 (1985).

23. H. J. Herrmann, A. Hansen, and S. Roux, Physical Review B 39(1), 637 (1989).

24. J. S. Przemieniecki, Theory of Matrix Structural Analysis (McGraw-Hill Book Company, New York, 1968). 